\title{
ERRATUM
}

\section{Inhibition of endothelial nitric oxide synthase activity and suppression of endothelium-dependent vasorelaxation by 1,2-naphthoquinone, a component of diesel exhaust particles}

\author{
Yang Sun · Keiko Taguchi $\cdot$ Daigo Sumi
}

Shigeru Yamano $\cdot$ Yoshito Kumagai

Published online: 5 July 2006

(C) Springer-Verlag 2006

Arch Toxicol (2005). DOI 10.1007/s00204-005-0043-3

At the final stage of typesetting the processing instruction "tgljoint @|Set Line Joint Style" found its way into the first sentence of the abstract. The publisher regrets this error.

The online version of the original article can be found at http://dx.doi.org/10.1007/s00204-005-0043-3

Y. Sun $\cdot$ K. Taguchi $\cdot$ D. Sumi $\cdot$ Y. Kumagai $(\bowtie)$

Department of Environmental Medicine,

Doctoral Programs in Medical Sciences,

Graduate School of Comprehensive Sciences,

University of Tsukuba, 1-1-1 Tennodai Tsukuba,

Ibaraki 305-8575, Japan

e-mail: yk-em-tu@md.tsukuba.ac.jp

S. Yamano

Faculty of Pharmaceutical Sciences, Fukuoka University,

8-19-1 Nanakuma, Jonan-ku, Fukuoka 814-0180, Japan

Y. Kumagai

Southern California Particle Center and Supersite,

Los Angeles, CA 90095-1772, USA 